\title{
LA INMERSIÓN EN UN PROCESO DURANTE TODO EL CURSO
}

\author{
INMERSING IN A PROCEDURE THROUGHTOUT A WHOLE YEAR
}

\author{
ANDREA SPADA JIMÉNEZ \\ Doctoranda área de Derecho Procesal \\ andrea.spada.j@hotmail.com \\ Universidad de Málaga
}

\section{Resumen:}

Es indiscutible la efectividad de la docencia práctica en el derecho procesal, por lo cual en el presente trabajo se expone un método docente que combina las diversas herramientas de aprendizaje práctico que tienden a utilizarse en la actualidad como complemento a la docencia. El modelo presenta una dinámica de trabajo grupal a llevar a cabo durante todo el curso con el planteamiento de casos prácticos que, a través de juegos de rol, se analizarán desde su fase preparatoria hasta su fase ejecutiva y se completará con otras actividades, con la intención de obtener unos resultados óptimos de aprendizaje.

Palabras clave: Derecho procesal, innovación docente, aprendizaje práctico, juegos de rol, tecnología, método docente, motivación.

\begin{abstract}
:
It is undeniable the effectiveness of practical teaching in Procedural Law, therefore a teaching method is set out in this paper which combines the diverse tools of learning by doing which currently tend to be used as a complement of teaching. The model presents the approach of a group work dynamic to carry out during the completely academic year, where would be analyzed cases studies through role-plays since pre-trial stage of proceedings until final judgment, with the intention to obtain optimal learning results.
\end{abstract}

Keywords: Procedural Law, teacher innovation, learning by doing, play of roles, technology, teaching method, motivation.

Sumario: 1. Introducción. 2. Desarrollo del método docente. 2.1. El primer día de clase. 2.2. Preparación de los contenidos. 2.3. Elección del caso judicial. 2.4. Inicio del procedimiento. 2.5. Visita a dependencias judiciales. 2.6. Asistencia a la celebración de vistas. 2.7. Juegos de rol para los actos procesales. 2.8. Sistema de evaluación periódica. 2.9. El uso de herramientas tecnológicas complementarias. 3. Resultados positivos pretendidos. 4. Conclusiones. Bibliografía.

REJIE Nueva época: Revista Jurídica de Investigación e Innovación Educativa

Núm.20, Junio 2019, pp. 31-47

[En línea] http://www.revistas.uma.es/index.php/rejie

Recibido: abril 2019

Aceptado: mayo 2019 


\section{Introducción.}

El estudio del derecho conlleva una tarea tanto teórica como práctica, siendo necesario el conocimiento desde ambas perspectivas para la consecución de un adecuado aprendizaje lo más realista posible, mediante el cual se sienten unas bases sólidas de formación para acceder al mercado laboral.

Ahora bien, para el aprendizaje del derecho procesal, más que para otro tipo de asignaturas de derecho sustantivo, es fundamental hacer hincapié en una metodología docente práctica porque su aplicación real es eminentemente práctica. Resultando mucho menos atractivo y funcional para un alumno el aprendizaje memorístico y a través de clases magistrales que uno basado en desarrollar las competencias que necesitan en torno a la aplicación y el funcionamiento de la Ley. No cabe duda de que para obtener dichas competencias es necesario ese conocimiento teórico, por lo que a través del presente trabajo se expondrá un modelo docente para obtener dichos conocimientos teóricos, pero, a través de la realización de actividades prácticas.

Para materializar lo expuesto, se modifica el método para la obtención de conocimientos teóricos en la consecución de un aprendizaje tan motivante como el que Edith STEIN describía de su profesor Adolf REINACH ${ }^{1}$, que sea profundo, interesante y lo más didáctico posible, en el que el docente sea el pilar de motivación para llevar a cabo todas las actividades del alumno.

Para la consecución de tal fin, se expone un método docente que resulta de la combinación de las diversas herramientas utilizadas hasta ahora en la docencia práctica del derecho procesal, pero contempladas desde una óptica global e integrada en un método práctico integral para una asignatura compleja como es derecho procesal. Dicho modelo consiste en una simulación a desarrollar durante todo el curso, otorgándole al alumno el papel de Letrado en todas las fases del proceso, a su vez se plantean actividades complementarias, como pueden ser visitas a las dependencias judiciales o realización de juegos en la red, acompañándose de evaluaciones periódicas para obtener resultados en cuanto a los conocimientos asimilados o la carencia de los mismos.

\section{Desarrollo del método docente.}

Hacer referencia a los juegos de roles, como son las simulaciones, utilizadas como una herramienta de aprendizaje no es algo novedoso, sin embargo, sí que es indiscutible su inclusión dentro del modelo de enseñanza del derecho procesal para que el alumno adquiera las capacidades básicas necesarias que le requiere el mundo laboral, por lo que considero que esta herramienta es imprescindible en un modelo docente, y por ende la base principal del presente.

Ahora bien, el método docente que se expondrá, se nutre de la aleación de los diversos complementos formativos docentes que se han planteado a través de la innovación, pretendiendo con ello crear un modelo docente actual, práctico y dinámico para el aprendizaje del derecho procesal, en el que no solo se aplique la simulación para el juicio oral stricto sensu o para una actuación concreta, sino que a lo largo de todo el curso los estudiantes se sumergirán en todo el proceso, combinando conocimientos teóricos y prácticos.

\footnotetext{
${ }^{1}$ VARGAS GÓMEZ-URRUTIA, M. y M. HERRERA MOLINA, P. (2019) “¿Qué podemos aprender sobre Edith STEIN sobre la investigación y la enseñanza del Derecho?” Diario La Ley, núm. 7931.
} 
Para desarrollarlo, por tanto, utilizaré técnicas de juegos, elaboración de esquemas, trabajo en grupo, actividades fuera del aula, juegos de rol y aplicaciones tecnológicas, con la intención de obtener una formación del alumno lo más completa posible.

La aplicación del método se prevé para las asignaturas de Derecho procesal, ya sean, derecho procesal civil como derecho procesal penal, como de aquellas otras concernientes a los procesos especiales, desarrollándose en cada una de ellas el proceso respectivo de estudio, comenzado por la fase preliminar y finalizando en la fase ejecutiva del mismo. Ahora bien, el presente modelo se plantea aplicado a la asignatura de Derecho Procesal Civil.

\subsection{El primer día de clase.}

Partiendo de lo anterior, el desarrollo de este método didáctico, comienza el primer día de clase con la proposición de un juego de preguntas y respuestas ${ }^{2}$, mediante el cual se divide la clase en grupos y cada uno de ellos recibe la misma cantidad de tarjetas que contienen preguntas cortas y concretas, relacionadas con el contenido que se impartirá en la clase, sobre todo el necesario como base del mismo, con el fin de valorar el nivel de conocimientos de los alumnos y así poder ajustar el desarrollo del curso. Ahora bien, los grupos que respondan las preguntas de forma favorable, dentro de un determinado periodo de tiempo, obtienen un punto y las que no puedan responderse o se hayan respondido de forma equivocada, pasarán por cada grupo hasta ser contestadas o descartadas, e igualmente cada una de ellas, se responderán por el docente y se incidirá en su comprensión con la intención de obtener una base preliminar para desarrollar el curso de la asignatura.

Con la elaboración del juego, el docente podrá observar los diferentes niveles de conocimientos de cada alumno, y con base en ello, podrá organizar a los estudiantes en los grupos en los que se desarrollarán las tareas a lo largo de todo el curso, dependiendo del fin pretendido y del grupo para dicha organización caben tres opciones:

La primera, que la división de los grupos se lleve a cabo teniendo en cuenta, la similitud en la base de conocimientos para que se desenvuelvan y evolucionen a lo largo del curso conforme a los mismos.

La segunda, organizar los grupos de una forma equitativa, es decir, llevar a cabo una distribución en la que cada grupo esté integrado por alumnos de diferentes niveles básicos de conocimientos, con el fin de crear un nivel de exigencia mayor de trabajo a los que tienen menos nivel para ajustarse a los otros y a la vez enriquecerlos o incentivarlos para que desarrollen la tarea de la forma más igualitaria posible.

La tercera, que la distribución de los grupos se desarrolle por los propios alumnos. Esta opción será la más acertada cuando la asignatura corresponde a los últimos cursos de la carrera, ya que con anterioridad se han relacionado entre ellos de forma grupal y se acomodarán de acuerdo con sus necesidades y preferencias, trabajando de una manera más cómoda.

\footnotetext{
${ }^{2}$ Siguiendo la línea docente propuesta por la autora acerca de comenzar el primer día de clase con juegos. KARINA CICERO, N. (2018) "El primer día de clases", Revista de Derecho y Educación. Education and Law review, núm. 18 [En línea] http://revistes.ub.edu/index.php/RED/article/view/22927.
} 


\subsection{Preparación de los contenidos.}

El segundo paso a llevar a cabo consiste en la elaboración de un esquema procesal que se entregará al alumno en torno a las fases del proceso, los pasos principales en los que se divide y las competencias de los órganos judiciales básicas, ello le permitirá familiarizarse con lo que va a descubrir a lo largo de todo el curso.

Dicho esquema es beneficioso tanto para los alumnos de primer curso que se enfrentan a la asignatura de derecho procesal sin tener una base de las asignaturas de derecho sustantivo, como para los alumnos de tercer curso, a los que les proporciona una actualización de conocimientos y una base del contenido de la asignatura.

Se debe tener en cuenta, que a pesar de que la asignatura en primer curso solo se centre en la configuración del proceso en general, se debería realizar igualmente de la materia a impartir durante todo el curso y de la configuración del proceso civil como en el penal, porque les facilitará organizar mentalmente los conocimientos.

En mi opinión, es fundamental ese primer contacto del alumno con el derecho procesal de una forma esquematizada, le permitirá saber dónde se inicia su aprendizaje y donde finalizará, obteniendo una visión general de la asignatura desde el principio, lo que le otorgará confianza, interés, seguridad y entusiasmo.

Seguidamente, una vez que se explica el esquema general de una forma totalmente asequible para su lenguaje, con el fin de familiarizarse con el mismo, y lo más importante, que comprendan lo que allí está expuesto ${ }^{3}$, empezaría el tercer paso o el inicio de la fase práctica. La cual, se organizaría con la división de la clase en grupos y a cada grupo se le asignarán dos casos prácticos, siendo lo ideal que en un principio sean sencillos y a medida que avance el curso, también se incrementará la dificultad de los mismos -se da por supuesto que los mismos se adecuarán al nivel y curso académico del alumnado- dilucidándose uno por la vía penal y otro por la civil para que lleven a cabo los pasos de todo el procedimiento.

\subsection{Elección del caso judicial.}

En cuanto a la elección de los supuestos objeto de análisis en el curso, caben varias posibilidades, puede ser el propio docente quien crea un caso judicial ficticio, adecuado al grado de sencillez o dificultad pretendido, se puede acceder a un expediente judicial o se puede hacer uso de los diversos medios multimedia existentes, ya sea el cine, la prensa o la novela.

Cabe recordar en este caso, que la implantación del contenido cinematográfico en la docencia adquiere cierta relevancia en España sobre todo en los últimos diez años, en los cuales diversas Universidades incluyen como cursos, seminarios o asignaturas optativas hasta crear varios Proyectos de Investigación ${ }^{4}$ llegando a formar parte de la Red de profesores para la Elaboración de Materiales Didácticos para la Enseñanza del Derecho a través del Cine.

\footnotetext{
${ }^{3}$ DONDI, A. / NIEVA FENOLL, J. (2017) "Diálogos sobre la docencia en derecho procesal (Dialogues about Teaching Procedural Law)”, Justicia: Revista de Derecho Procesal. J.B. Bosch, núm. 2, págs. 81116.

${ }^{4}$ Universidad Juan Carlos de Madrid, Barcelona. La Coruña, Oviedo, Burgos, entre otras. Con respecto a ello: ÁLVAREZ GONZÁLEZ, E.M. (2015), "Una experiencia docente aplicada en la asignatura Fundamentos de Derecho Público del grado en Criminología", DEL VALLE MEJIAS M.E. (Coord.): Experiencias en docencia superior. Madrid: ACCI.
} 
Uno de esos proyectos de investigación, concretamente el Proyecto DeCine, mediante la creación de una página web, ha contribuido a facilitar la tarea docente mediante la elaboración de un catálogo de películas adecuadas para su uso didáctico y clasificadas en relación con las diferentes ramas del derecho, donde en las mismas se describen y comentan las actividades que se pueden realizar por el alumno, a través de la elaboración de una ficha técnica docente que así lo incorpora ${ }^{5}$.

Por lo cual, como punto de partida de la tarea propuesta, se puede acceder a la página $w b^{6}$ perteneciente a dicha colaboración para obtener el contenido de una película y basar el caso en ella, aunque la misma está pensada para el uso de esta herramienta docente de forma autónoma, es decir para actividades más extensas, concernientes a temas concretos y no solo como la base de un supuesto.

Se puede utilizar la lectura de prensa, como base para el caso judicial, mediante la cual, a su vez, se promueve el pensamiento en torno a su "traducción jurídica", el reconocimiento de figuras teóricas en la realidad, la asimilación de conocimientos y las diversas perspectivas con las que se puede analizar una noticia.

Cabe también, el uso de la lectura de novelas específicamente seleccionadas, en las que se desarrolla la trama de un suceso penal, como si de un caso real se tratara para un posterior desarrollo por parte del alumno, como sería interponer una querella. Aunque no solo se suelen utilizar las novelas para ello, sirven como base para el debate o para la elaboración de escritos de calificación ${ }^{7}$, el problema de ello es que se corre el riesgo de desmotivar al alumno por añadirle esa carga lectora y el resultado puede ser que solo lo realicen algunos participantes del grupo o que se obtenga un resumen de la misma a través de la red.

Destacado es, que la utilización de los medios multimedia, junto con la elaboración actividades concretas adecuadas para los estudiantes les ayuda a reflexionar, a ser críticos, al planteamiento de diversos comportamientos en situaciones moralmente comprometidas, lo que resulta aplicable e interesante para la impartición de asignaturas como Ética o Filosofía del Derecho ${ }^{8}$.

En cuanto al derecho procesal concretamente, les puede facilitar la comprensión de los principios y la forma del proceso, siempre y cuando la película se encuentre basada en la propia realidad jurídica y les haga reflexionar concretamente sobre dichos aspectos jurídicos, con lo que son actividades que deberían elaborarse en el aula con un seguimiento por parte del docente a fin de obtener la adquisición de los conocimientos pretendidos de ello'.

\footnotetext{
${ }^{5}$ En relación al funcionamiento de la Red: FERRERO BAAMONDE, X. / PÉREZ-CRUZ MARTÍN, A.J. (2011) "El cine como instrumento metodológico en la docencia del Derecho Procesal", PICÓ I JUNOI, J. (Dir.) Aprendizaje del Derecho Procesal: Nuevos retos de la enseñanza universitaria. Barcelona: J. M. Bosch.

${ }^{6}$ Proyecto DeCine de la Universidad de A Coruña: https://proyectodecine.wordpress.com/

${ }^{7}$ SAIZ GARITAONANDIA, A. (2011), "Caminando hacia el aprendizaje cooperativo en la docencia del Derecho Procesal Penal”, UNIVEST. Institut de Ciències de l'Educació Josep Pallach (ICE). [En línea] https://dugi-doc.udg.edu/handle/10256/3695

${ }^{8}$ Método didáctico para abordar el tema de la igualdad: CENTENERA SÁNCHEZ-SECO, F. (2017), "Clara Campoamor y el valor de la Igualdad: Una propuesta docente", Revista Jurídica de Investigación e Innovación Educativa (REJIE), núm 16, págs. 11-33 [En línea] http://www.eumed.net/rev/rejie

${ }^{9}$ Ejemplo de la actividad conducente a la obtención de conocimientos en cuanto a la forma y principios del proceso: CUBILLO LÓPEZ, I.J. / FERNÁNDEZ CARRIÓN, C. / DE PRADA RODRIGUEZ, M. (2011), "Utilización del cine como herramienta para la docencia del Derecho Procesal", PICÓ I JUNOI,
} 
Ahora bien, estas actividades no constituyen una forma de aproximación del alumno a la realidad jurídica, sino más bien que facilitan el desarrollo de habilidades en torno a la crítica jurídica o para mejorar la expresión escrita, si se acompañan de resúmenes o informes, sin embargo, para ello será necesario que dicha actividad se lleve a cabo con bastante asiduidad, no basta con una o dos sesiones para adquirir dichas competencias, lo que reduciría el tiempo para impartir el resto de contenido de la asignatura.

A su vez, atendiendo al punto de vista del alumno, el desarrollo de dichas actividades, acompañadas de la elaboración de resúmenes o informes jurídicos, parece que no resultan de interés para ellos como la realización de otras actividades ${ }^{10}$, por lo que se corre el riesgo de obtener un efecto contrario al pretendido.

Por todo lo cual, y teniendo en cuenta la necesidad de maximizar todo el tiempo posible para la impartición de la completa programación docente, en mi opinión, estas actividades no resultan ser tan rentables como otras herramientas de aprendizaje, teniendo en cuenta el tiempo invertido y el resultado obtenido, por ende, no serán incluidas dentro del presente método docente.

Por ello, aunque quedará al arbitrio de cada docente la elección del método, en este planteamiento considero que lo más recomendable sería poder obtener un expediente judicial completo que ya se hubiera sentenciado y ejecutado, entendiendo que se podrá seguir paso a paso el procedimiento y finalmente comparar actuaciones reales y simuladas en el aula sirviendo de aprendizaje y motivación para el alumno al encontrarse en una posición próxima a la realidad forense.

\subsection{Inicio del procedimiento.}

Una vez que se hace entrega del supuesto civil se procederá a la elaboración de la demanda en la propia aula, para así, poder solventar problemas de interpretación legislativa, añadir los conocimientos teóricos necesarios para elaborar dicha tarea y apoyar al desarrollo de la misma con la implicación de todos los integrantes del grupo, evitando que solo unos pocos realicen el trabajo.

Como es sabido, a través de esta tarea se podrán analizar los temas de competencia, jurisdicción y sus conflictos, la acción, las partes del proceso, entre otras.

El siguiente paso, consistirá por tanto en la entrega de la demanda al docente, cuyo rol será "el del juzgado que por turno corresponda", a través de la plataforma Moodle de la Universidad utilizada, de la que recibirá la resolución en torno a su admisión o no en la siguiente sesión, con lo que obtendrán los conocimientos en torno a las manifestaciones procesales del principio dispositivo, los presupuestos procesales, la admisión de medidas cautelares solicitadas, las diligencias de investigación, entre otras, y si el supuesto lo hemos obtenido de un expediente judicial, se podrá entregar la admisión o inadmisión real del órgano judicial, lo que les permite observar el formato real del documento.

Si se procede a una inadmisión deberá ser analizada en clase y solventada a través de la subsanación correspondiente, como si el Letrado de la Administración de Justicia le

J. (Dir.) Aprendizaje del Derecho Procesal: Nuevos retos de la enseñanza universitaria. Barcelona: J. M. Bosch.

${ }^{10}$ En este sentido, los alumnos encuestados en el estudio llevado a cabo: GUTIERREZ BERLINCHES, A. / DE PRADA RODRIGUEZ, M. / CUBILLO LÓPEZ, I.J. (2010) "Las visitas a Juzgados como actividad práctica para la docencia del Derecho Procesal", Revista Jurídica de Investigación e Innovación Educativa (REJIE), núm. 2, págs. 111-122 [En línea] http://www.eumed.net/rev/rejie 
hubiera requerido su subsanación, también se procederá a la interposición del recurso por una inadmisión, para poder englobar todas las actuaciones procesales dentro del procedimiento.

\subsection{Visita a dependencias judiciales.}

Para ganar tiempo por parte del docente en su rol de actuación como juzgado se puede plantear la realización de la visita a las dependencias judiciales, no a las celebraciones de vistas, sino al espacio físico, aproximando al alumno a la realidad judicial, y utilizando dicha actividad para el tema concerniente al personal de la administración de justicia. Otra actividad complementaria, en relación con ese tema, consiste en invitar a personal perteneciente a la misma, como puede ser un policía judicial y que explique la consistencia de su labor y como se configura.

La asistencia a la propia dependencia judicial, permite conocer el funcionamiento de la institución, la distribución del personal y las competencias inherentes a cada uno de ellos, les acerca a la realidad, de modo que con ello obtienen motivación, les ayuda a ganar confianza con el docente, dado que tienen una actividad fuera de lo común, a su vez, les facilita la incorporación de conocimientos sobre las administración de justicia asimilando la experiencia cuando se lleve a cabo el estudio teórico ${ }^{11}$, con lo cual la explicación teórica se podrá reducir, permitiendo ocupar el espacio en temas con mayor relevancia en la práctica real ${ }^{12}$.

Consecuencia favorable de tal actividad, es que, al familiarizarse con el espacio físico de la administración de justicia, les impulsará a acudir por motu proprio a observar vistas orales y a inmiscuirse en el mundo judicial real, dotando así de dinamismo el estudio del derecho procesal, que quizás de una manera clásica, se obtiene de una forma más tediosa.

Posteriormente, y siguiendo con el modelo expuesto, ya habiendo entregado las resoluciones de admisión o inadmisión a los alumnos, en la búsqueda del aprendizaje de los recursos, el docente podría escoger por ejemplo, un caso de litisconsorcio, para obtener el aprendizaje del alumno en cada caso concreto, debatir acerca de las tácticas de defensa, la elaboración de las demandas que se han realizado por parte de todos los de la clase, obteniendo siempre una dinámica de grupo en la que el profesor se encuentra inmiscuido en el mismo.

En este punto, sería recomendable aplicar un cambio de rol de los grupos, entregando a cada uno de ellos las demandas interpuestas por sus compañeros para la correspondiente contestación de la misma, facilitando el desarrollo de competencias necesarias para el ejercicio de la abogacía.

\subsection{Asistencia a la celebración de vistas.}

Una forma eficaz de aproximar a los estudiantes a la realidad práctica jurídica y de asentar los conocimientos teóricos adquiridos, es a través de la asistencia a las salas

\footnotetext{
11 Sobre este particular: GONZALES PILLADO, E. (2009), "Una experiencia en la docencia del practicum de Derecho Procesal", Revista de Formación e Innovación Educativa Universitaria. Vol. 2, núm. 3, págs. 151-159.

12 Basado en la propuesta expuesta en: DE MIRANDA VAZQUEZ, C. (2015). "Propuesta de modelo para la docencia del Derecho Procesal", Revista de Educación y Derecho. Education and Law Review , núm. 12, págs. 151-167.
} 
judiciales, ya sea pudiendo asistir a la celebración de cualquier tipo de vista, o ya sea a la propia dependencia judicial.

En relación con la asistencia a la celebración de las vistas en las salas judiciales, en el que el alumno ostenta el papel de espectador de una vista real, no cabe duda de su efectividad práctica como complemento de la docencia del derecho procesal ${ }^{13}$, ya que por un lado adquiere conocimientos en torno al lenguaje, las posturas, la forma en la que dirigirse al órgano judicial y el turno de palabra de las partes, es decir obtiene conocimientos formales necesarios para la práctica forense, y por otro, le facilita el asimilar conceptos teóricos, ahora bien, en cuanto a la adquisición de estos conocimientos de fondo, con la simple asistencia a la vista no se puede pretender dicho objetivo, sino que se deberá acompañar del material necesario previo de todo el proceso y de un apoyo por parte del docente o de las partes intervienes para su explicación.

Ahora bien, a pesar de los beneficios que se extraen de dicha asistencia existen varios inconvenientes en el desarrollo de dicha tarea, principalmente los siguientes:

- La necesidad de obtención de todo el material referente al proceso por el cual se celebra la vista, ya que para obtener unos conocimientos completos en la experiencia, tanto los conocimientos sustantivos y los formales sobre la actuación en la misma, se deben complementar con la justificación de dichas actuaciones, por lo que se hará necesaria la complicidad de forma altruista del personal de la administración de justicia con la que obtener la documentación del proceso y una explicación del desarrollo de la vista al principio y al final de la celebración.

- La dimensión de las salas judiciales tiene una capacidad limitada e inferior al número correspondiente de estudiantes que componen el aula, con lo que habrá que hacerlo por turnos y dividirlos por lo que observarían cuestiones de diversa índole entre ellos y generaría una pérdida de tiempo.

- Las situaciones imprevistas en las sedes judiciales como suspensiones, aplazamientos y conformaciones conllevan la imposibilidad de elaborar la actividad que estaba prevista dificultando la obtención de formación que se pretendía con ellas.

- La dificultad en la coordinación entre la asistencia de los alumnos a las salas judiciales, con la docencia y los órganos judiciales, sobre todo en aquellas zonas donde los organismos judiciales y las universidades no se encuentran ubicadas en cercanía.

Partiendo de ello, se han buscado otras vías en las que se pudieran obtener los mismos beneficios que la asistencia en sala judicial, encontrándose, la celebración de juicios en aulas judiciales incluidas en la propia Universidad, las grabaciones de vistas reproducidas en el aula y las simulaciones, sin embargo, con esta última no se perciben los mismos resultados ya que los estudiantes son los propios actores y los que manejan el curso del procedimiento, con lo cual la obtención de conocimientos en ambas situaciones, aunque efectiva, es distinta.

En lo referido a la existencia de aulas judiciales en la propia Universidad, es una solución factible, útil y eficaz a los problemas planteados, reflejándose ello en la

\footnotetext{
${ }^{13}$ En este sentido, a través de las encuestas elaboradas a los alumnos: GUTIERREZ BERLINCHES, A. / DE PRADA RODRIGUEZ, M. / CUBILLO LÓPEZ, I.J. (2010) "Las visitas a Juzgados como actividad práctica para la docencia del Derecho Procesal”, Revista Jurídica de Investigación e Innovación Educativa (REJIE), núm.2, págs. 111-122 [En línea] http://www.eumed.net/rev/rejie
} 
utilización del aula judicial de la Facultad de Derecho de la Universidad de Málaga a través del Convenio de Colaboración con el Consejo General del Poder Judicial (Tribunal superior de Justicia de Andalucía) y la Universidad de Málaga (Facultad de derecho) firmado el 18 de abril de 2001, mediante el cual se pone a disposición el aula judicial para la celebración de juicios reales y en la que los actores de los mismos, con su aceptación previa, desarrollan la vista real pero de una forma más didáctica, mediante la cual, los estudiantes reciben toda la documentación referente al proceso con anterioridad a la misma, se realizan explicaciones en cuanto a estrategias de defensa utilizadas por las propias partes que concurren en el proceso y cuando finaliza la vista se les otorga un espacio referente a cuestiones del proceso. Por lo cual, gracias a la colaboración expuesta en dicho Convenio se han resuelto los inconvenientes planteados en un principio y se obtiene una ventaja añadida en cuanto a la implicación de los estudiantes, y es que los mismos tienden a ser más activos cuando se encuentran en un entorno familiar que en el judicial, por lo que obtienen un aprendizaje más completo con la realización de la actividad ${ }^{14}$.

Otra forma de solventar los inconvenientes de la asistencia a las vistas de las salas judiciales, se ha llevado a cabo con la utilización de medios técnicos audiovisuales para la grabación de vistas y reproducción en el aula, siendo la Facultad de Derecho de la Universidad de Granada, la que desarrolla tal iniciativa de forma completa a través de un proyecto de innovación docente que ha llevado a cabo el Departamento de Derecho del Trabajo y de la Seguridad Social denominado "Realidad jurídico laboral en el aula: Guías de trabajo autónomo con medios audiovisuales", con el fin de obtener un conocimiento completo del derecho procesal laboral mediante el mismo, ya que en las grabaciones se incluyeron tanto las formas judiciales como las extrajudiciales de solución de conflictos, pero a su vez dichas grabaciones se acompañaban de un material didáctico con la documentación referente a los mismos y la explicación de cada categoría de las expuestas, con lo que se obtiene una visión completa de lo que conforma el derecho procesal laboral, adquiriendo el estudiante formación formal y sustantivo del contenido del proceso ${ }^{15}$.

La actividad llevada a cabo por la Universidad de Granada es otra solución factible y efectiva, que solventa los problemas derivados de la asistencia física a la propia sala judicial y la gran ventaja es que se recrean todas las situaciones procesales que pudieran suceder independientemente del calendario judicial, lo que permite su utilización en el momento que se requiera oportuno pudiendo sustituir las lecciones magistrales en cualquier caso, aunque sí que requiere de una labor meritoria por parte de todos los sujetos intervinientes, por lo que para los docentes de derecho procesal laboral será de agradecer que todo el material se encuentre publicado y disponible para su utilización por cualquier interesado, aunque lo ideal sería que se pudiera poner en práctica para la enseñanza del derecho procesal en cualquiera de sus jurisdicciones a través de la cooperación docente.

\footnotetext{
14 DE LUCCHI LÓPEZ-TAPIA, Y. / ROBLES GARZÓN, J. A. (2011) "Experiencias desde el aula judicial de la Facultad de Derecho de la Universidad de Málaga”. PICÓ I JUNOI, J. (Dir.) Aprendizaje del Derecho Procesal: Nuevos retos de la enseñanza universitaria. Barcelona: J. M. Bosch.

${ }^{15}$ MOYA AMADOR, A. / SERRANO FALCÓN, C. /TOMÁS JIMÉNEZ, N. "Realidad jurídico laboral en el aula" (2011). ARBOR Ciencia, Pensamiento y Cultura, Vol. 187 - Extra 3, pp. 273-277 [En línea] http://arbor.revistas.csic.es/index.php/arbor/article/view/1439/1447
} 
En la búsqueda de otras alternativas sustitutivas a la presencia física en las sedes judiciales, se encuentra la que se han plasmado en la Universidad de País Vasco ${ }^{16}$, donde el modelo docente aplicado, incluía una actividad de tres sesiones que se ha podido desarrollar a través de una complicidad encomiable por parte del Presidente de la Audiencia Provincial de Vizcaya, dado que el mismo le entregaba al docente las grabaciones de las vistas celebradas junto con los escritos de calificación presentados por las partes, con lo cual se podían reproducir dichas vistas en el aula, acompañadas de las explicaciones necesarias del docente, adquiriendo por tanto los estudiantes no solo las cuestiones formales de actuación en sala, sino también las cuestiones procesales en ella suscitadas y la elaboración de escritos de calificación, concretamente en el desarrollo de dicha tarea también se incluyó el escrito de la Sentencia por parte de los alumnos en grupo y el posterior debate sobre ello, lo cual también favoreció al desarrollo de las habilidades de trabajo cooperativas y a desenvolverse con el lenguaje oral en el aula.

En las tres actividades expuestas se consigue la finalidad del objetivo pretendido en cuanto al papel del alumno como un espectador de la vista, pero a su vez adquiriendo los conocimientos teóricos que engloban dicha actividad, como si estuviera en la propia sede judicial, pero estando en el aula y solventando los problemas materiales que ello conlleva, facilitando la labor docente y de aprendizaje, obteniendo una formación completa y más dinámica del derecho procesal, lo que determina la necesidad de la inclusión dentro del modelo docente pretendido.

Por todo lo expuesto, cuando nos adentremos en la fase de juicio oral, lo más adecuado sería acudir a la celebración de vistas o si no es posible, acceder a los medios sustitutivos que se han plasmado en el presente trabajo para que puedan tener una visión de la configuración de la fase del juicio oral en sentido estricto, siendo ésta posteriormente interpretada a través de simulaciones, que en lo posible se desarrollarán en el Aula Judicial de la Facultad.

\subsection{Juegos de rol para los actos procesales.}

A pesar de que el alumno se encontrará inmiscuido dentro del proceso a lo largo de todo el curso, por lo que su simulación se desarrollará todo el tiempo, esta fase del modelo, en concreto, está diseñada para cada una de las actuaciones procesales que se pueden celebrar en forma de vista, como pueden ser una audiencia a las partes derivada de la solicitud de medidas cautelares o la audiencia previa al juicio, y por supuesto, el propio juicio oral stricto sensu.

La forma en la que se debería desarrollar la simulación puede ser diversa y dependerá de varios factores, desde la organización y el tamaño del grupo, hasta la disciplina con la que se realiza la actividad, en este caso, de acuerdo con la distribución de los casos en grupos para la interposición de demanda y el cambio de rol que se realiza para la contestación de la misma, cabe hacer la simulación dividiendo a unos grupos como demandantes y a otros como demandados, siendo el docente el que así lo decida.

El papel del docente en estas actividades, puede ser como espectador o como actor, aunque, sí que se aboga por que las mismas se desarrollen de forma totalmente

16 SAIZ GARITAONANDIA, A. "Caminando hacia el aprendizaje cooperativo en la docencia del Derecho Procesal Penal” (2011), UNIVEST. Institut de Ciències de l'Educació Josep Pallach (ICE). [En línea] https://dugi-doc.udg.edu/handle/10256/3695 
autónoma por los alumnos ${ }^{17}$, adquiriendo el docente un papel de espectador durante la actividad, en el modelo que aquí se expone, el docente tiene el papel de juez desde el principio, por lo que lo adecuado sería que continúe con dicho rol, sobre todo para darle sentido al proceso que se está desarrollando.

Una vez finalizada la vista simulada, el docente deberá corregir los errores que hubiera percibido y ser un apoyo fundamental en dicha formación, así como realizar diferentes planteamientos a los expuestos con el fin de incentivar a la investigación y adquisición de conocimientos.

En cuanto al espacio físico en el cual se puede desarrollar la simulación de una vista, lo ideal sería ejecutarla en un aula judicial de la propia Universidad, sería la forma más adecuada y cómoda para crear el ambiente pretendido y facilitar la adquisición de los conocimientos formales, ahora bien, de no caber tal posibilidad, se deberá recrear en el aula de clase una especie de sala judicial en la que se respete la forma y las posiciones de los actores de la vista.

Se puede tener en cuenta que el planteamiento de esta actividad, también se puede realizar de forma interdisciplinar, es decir, en la que colaboren docentes de más de una disciplina para la elaboración de la simulación, ahora bien, dicha colaboración resultará una tarea de consistente carga laboral teniendo en cuenta toda la organización de la tarea en cooperación de más de un docente para la obtención del objetivo pretendido con la misma, sin embargo gracias a la labor que ha desarrollado un grupo de docentes de la Universidad de Málaga es posible acceder a una guía consistente en diez puntos de planificación y desarrollo de dicha tarea, lo cual facilitará dicha tarea para cualquier docente que considere su planteamiento ${ }^{18}$.

Finalmente, en relación con las simulaciones, como forma de motivación para el alumno, se puede plantear la posibilidad de inscripción en las simulaciones configuradas en forma de competición, las denominadas Moot Court Competition, las cuales son celebradas en las Universidades y en las propias sedes de Tribunales Internacionales con lo que se da un salto cualitativo en el aprendizaje del derecho en un entorno cooperativo y cosmopolita ${ }^{19}$.

${ }^{17}$ MORA CAPITÁN, B. (2011). "El aprendizaje del Derecho Procesal mediante simulación de juicios", PICÓ I JUNOI, J. (Dir.) Aprendizaje del Derecho Procesal: Nuevos retos de la enseñanza universitaria. Barcelona: J. M. Bosch.

18 GARCÍA MAGNA, D., CASTILlO RODRÍGUEZ, C., RIOS MOYANO, S., CRISTOFOL RODRÍGUEZ, C., CARRASCO SANTOS, M., RODRÍGUEZ MÉRIDA, R., GONZÁLEZ RAMÍREZ, D., PASTOR GARCÍA, I., \& GONZÁLEZ RAMÍREZ, D. (2011). "La interdisciplinariedad en la educación superior: propuesta de una guía para el diseño de juegos de rol". Education In The Knowledge Society (EKS), 12 (1), págs. 386-413. [En línea]

http://revistas.usal.es/index.php/eks/article/view/7894/7937

${ }^{19}$ Actualmente, existen bastantes competiciones que abarcan diversas materias de derecho internacional y europeo, desde el derecho tributario hasta penal, siendo los más destacables, los siguientes:

- El International Criminal Court. Moot Court Competition, el cual simula un proceso ante la Corte Penal Internacional (CPI) cuyo juicio se celebra en La Haya en las propias salas de la CPI en la que participan cincuenta y cinco países durante el 2019, entre los cuales, lamentablemente España no se encuentra incluida. [En línea] http://iccmoot.com/

- En la misma línea se celebra el Nuremberg Moot Court Competition, donde se simula un juicio de derecho penal internacional en la propia sala de vistas 600 del Palacio de Justicia de Núremberg. [En línea] https://www.nuremberg-moot.de/index.php?id=283

- La asociación The European Law Students' Association (ELSA) también organiza la John H Jackson Moot, mediante la cual se realiza una simulación en cuanto al procedimiento de resolución de conflictos de la Organización Mundial del Comercio, con el fin de desarrollar conocimientos en torno 
Estas actividades, son organizadas por asociaciones en colaboración con las universidades y organismos internacionales, mediante las cuales se elabora un expediente judicial ficticio accesible para los participantes en la propia página web oficial y con el que se enfrentarán a las fases del procedimiento tanto escritas como orales divididas en diversas rondas que se celebran en varios Estados, exceptuando la ronda final que se celebra en la sede concreta que establezca la competición.

\subsection{Sistema de evaluación periódica.}

Para afianzar todos los conocimientos que han sido adquiridos de la forma expuesta, en el presente modelo que planteo, me parece interesante que se realice una evaluación periódica concerniente en un examen de tipo test cada tres semanas.

La evaluación se puede desarrollar a través de las aulas TIC de la Facultad, utilizando la plataforma Moodle de la Universidad, lo que resulta más fácil y práctico para corregir y establecer un tiempo determinado de respuesta, o se puede realizar en el aula de una forma tradicional.

Las preguntas que incluirá la evaluación serán acordes con las actividades que se hayan realizado, si acaban de iniciar el procedimiento, no se les podrá preguntar por la sentencia. Consistirán, sobre todo, en casos prácticos pequeños y en las mismas se permitirá el uso de la legislación, pero necesitarán un estudio previo para poder responder en el tiempo previsto, así se ven obligados a seguir un estudio de la asignatura a diario. Ejemplos genéricos de formulación de preguntas, podrían ser los siguientes: Juan, nacional español comete un delito contra la salud pública en Argentina, sin embargo, allí no se ha incoado procedimiento. ¿Tiene competencia el órgano judicial penal para incoar el procedimiento? O, Juan, arrendador, interpone demanda por impago de renta por parte del arrendatario. Determine la competencia objetiva y territorial en el presente caso.

La presente evaluación deberá ser aprobada como requisito preceptivo para presentarse a examen, ya que, con ello se consigue favorecer en la preparación del alumno en torno al formato y tipo de preguntas del examen final al que se enfrentará y al estudio de forma continua de la asignatura.

al derecho mercantil internacional y cuya final se celebra en Ginebra. [En línea] https://johnhjacksonmoot.elsa.org/about/

- En consonancia con la resolución de conflictos comerciales internacionales a través del arbitraje, se celebra la competición The Annual Willem C. Vis International Commercial Arbitration Moot en Viena. [En línea] https://vismoot.pace.edu/site/scholarships

- En relación con simulaciones ante la Corte Internacional de Justicia en torno a materia de derecho internacional, The International Law Students' Association (ILSA) organiza la Philip C. Jessup International Law Moot Court Competition, siendo una asociación a nivel internacional, la competición en simulaciones es la que tiene mayor participación y prestigio celebrando una ronda final de duración semanal en Washington, D.C. [En línea] https://www.ilsa.org/

- En materia de tributación internacional el Observatorio Iberoamericano de Tributación Internacional desarrolla una simulación internacional denominada Iberoamerican Tax Moot Court Competition, que se celebra cada año en una Universidad diferente. [En línea] http://oiti.org/

- En cuanto al derecho europeo, a través de la European Human Rights Moot Court Competition que organiza ELSA, se realiza una simulación de un procedimiento ante el Tribunal Europeo de Derechos Humanos, siendo la final en Estrasburgo en la propia sede del mismo. [En línea] https://ehrmcc.elsa.org/

- En España, se celebra Moot Madrid. Competición Internacional de Arbitraje y Derecho Mercantil, desde 2009, donde se simulan casos concernientes al derecho mercantil internacional cuya resolución se ejecuta a través del Arbitraje Mercantil Internacional. [En línea] http://www.mootmadrid.es/2019/ 
Por otro lado, con este sistema, también se beneficiará el docente como forma auto evaluativa, para observar si su explicación y su método están dando resultados y en donde se encuentran las carencias de aprendizaje.

La evaluación final, en mi opinión, debería consistir en la resolución de varios casos prácticos pequeños en sintonía con los exámenes periódicos y uno o dos casos prácticos más amplios, acompañado de la legislación, con lo que se consigue que el alumno pueda desarrollar todas las competencias adquiridas a lo largo del curso. Un ejemplo de los casos prácticos más amplios sería la elaboración de una demanda para un caso concreto o la interposición de un recurso contra la sentencia de otro caso, en la cual debe demostrar el conocimiento de varios temas para poder aplicar la legislación de forma correcta.

\subsection{El uso de herramientas tecnológicas complementarias.}

A los instrumentos metodológicos docentes clásicos se han incluido factores tecnológicos, desarrollándose así una tarea docente más dinámica o sustitutiva de la realidad, con la que se facilita el aprendizaje de los alumnos, lo que no significa que se facilite también para el docente, ya que, en cada actividad virtual, se encuentra una labor significativa por el docente en su configuración.

La utilización de las plataformas digitales, como las plataformas Moodle, no resulta ser algo novedoso, siendo utilizadas diariamente en todas las Universidades españolas, tanto para los estudios presenciales, como para los que se llevan a cabo a distancia, permitiendo el desarrollo de una labor más personalizada y con mayor implicación por parte del alumno y del docente.

Ahora bien, para aquellos estudiantes a distancia, los cuales tienen dificultades en torno a la obtención de conocimientos prácticos, la utilización de plataformas virtuales les ha brindado la posibilidad de adquirir las competencias que solo las simulaciones o la asistencia a juicios las podrían facilitar.

Es en la Universidad a distancia de Madrid, donde un grupo docente ha desarrollado como complemento formativo de las asignaturas Derecho de Obligaciones y Derecho de los Contratos $^{20}$, simulaciones a través de la red, utilizado la plataforma virtual de la Universidad para la fase preparatoria de la vista y el programa Second Life para la celebración de la misma. Ese programa permite recrear cualquier actividad que se desee de forma virtual, el espacio físico y las personas que actúan en el mismo a través de avatares, pudiendo comunicarse entre ellas en tiempo real, lo que permite obtener las ventajas de la realidad desde cualquier ordenador, sin necesidad de un traslado físico y una coordinación temporal dificultosa.

Claramente, dicha actividad no puede sustituir el aprendizaje obtenido por la asistencia a salas judiciales reales, pero sí que, para los estudios a distancia, sirve para sustituir los juegos de rol que los alumnos realizan de forma presencial con lo que es una gran herramienta complementaria a tener en cuenta para la docencia del derecho procesal a distancia.

Ahora bien, los docentes de un sistema presencial no necesitan incluir la tecnología 3D en su modelo docente, más que como forma de entretenimiento educativo, pero sí que

20 MONTERROSO CASADO, E. / ESCUTIA ROMERO, R. "La enseñanza práctica en 3D: Juicio virtual”, @tic. revista d'innovació educativa, núm. 6, 2011, págs. 46-54. [En línea] https://www.redalyc.org/articulo.oa?id=349532302006. 
sería bueno, en mi opinión, fomentar la colaboración virtual entre los alumnos para las diversas actividades de preparación o resolución del juicio oral de un procedimiento, utilizando la plataforma de Google Docs. En vistas del mercado laboral actual, multidisciplinar y colaborativo, es favorable el desarrollo de actitudes cooperativas por vías telemáticas, externas a las plataformas universitarias, porque seguramente lo utilizarán en un futuro.

La posibilidad de asistir a juicios orales se ve mermada como ya hemos visto por diversos factores externos al docente o al alumnado, por lo que el uso de la red también nos puede beneficiar en ello.

Plataformas virtuales como YouTube permiten poder presenciar juicios orales mediáticos, porque son reproducidos en streaming por los propios medios de prensa, con lo cual, podemos acceder a ellos para poder explicar de forma virtual las actuaciones procesales y las tácticas de defensa utilizadas. Por otro lado, cabe el planteamiento en el que el docente acuda a la vista que considere oportuna para incluir en el aprendizaje, subirla a la plataforma y poder comentar en el aula las actuaciones relevantes, siempre y cuando las partes estén de acuerdo en ello, protegiendo la intimidad de las mismas.

Finalmente, para completar el presente modelo, se plantea la utilización de las aplicaciones de Kahooth o Socrative, siendo juegos de preguntas y respuestas elaboradas por el propio docente, mediante las cuales los estudiantes asimilan los conocimientos de clase a la vez que se divierten.

Las preguntas que se pueden plantear en estos juegos son cortas, por lo que cabrían las del siguiente tipo: ¿Quién tiene la competencia para enjuiciar delitos con penas de 10 años? Y en las multirespuestas establecemos los diversos órganos judiciales y una de ellas será la correcta.

El desarrollo del juego de preguntas a través de las presentes herramientas no conlleva la inversión de un tiempo excesivo por parte del docente por lo que pueden ayudar a plantearse al final de cada lección, ya que para los alumnos resulta de gran entretenimiento por el formato de aplicación móvil.

\section{Resultados positivos pretendidos.}

- La motivación, el interés y la dinámica de grupo que se creará con el método planteado será muy beneficioso para asimilar e interiorizar los conocimientos de una disciplina cuya aplicación real es inminentemente práctica, por lo que su aprendizaje también debe serlo.

- El desarrollo de la tarea con la obtención de expedientes judiciales reales conllevará a que se familiaricen con el formato y el lenguaje de los escritos que recibirán por parte de un órgano judicial.

- Con la simulación de las vistas, adquieren los conocimientos formales que acompañados del resto de actividades permiten el desarrollo competencias, cognitivas, competitivas y lingüísticas.

- Los estudiantes adquieren los conocimientos en torno al funcionamiento de la administración de justicia y el espacio físico judicial donde se desarrollará su labor en el futuro.

- Complementario a lo anterior y con suma importancia, en mi opinión, los alumnos aprenderán a dominar el uso de la norma para su aplicabilidad práctica, 
es importante que desde un principio adquieran las facultades en torno a la utilización de la legislación como base para el estudio.

- En cuanto al aprendizaje dividido en grupos y de forma conjunta, permite desarrollar competencias cooperativas, el intercambio de opiniones entre ellos, el respeto y la transferencia de conocimientos y sobre todo la creación de una dinámica grupal de la clase, lo que se traduce en el interés por asistir a las clases y participar en las actividades.

\section{Conclusiones.}

Con el método planteado, el alumno se inmiscuye dentro de una actuación en la que desarrolla un papel, adquiriendo la posición de actor de la tarea que se le encomiende con base en un supuesto judicial, lo que conlleva que solo por el hecho de tener que actuar en público, -en frente de sus compañeros y docente- él mismo se esfuerza en aplicar sus conocimientos teóricos de manera práctica, a la vez que está desarrollando diversas facultades que preceden al ejercicio de la profesión, siendo ese el objetivo donde se debe focalizar la enseñanza del derecho procesal.

El alumno desarrollará las competencias formales y específicas en torno a las actuaciones en sala, como son, el comportamiento, la oratoria y la expresión de un lenguaje jurídico, a la vez que interiorizará los conocimientos teóricos, no solo en torno a las fases del proceso y las actuaciones procesales, sino también en torno a la materia objeto del proceso, con lo que obtiene un aprendizaje completo, dinámico y más cercano a la realidad que en comparación con un método docente tradicional.

Con aplicación o no del método expuesto, la innovación docente no solo en el derecho procesal sino en cualquier rama del derecho se encuentra latente, se han planteado diversas herramientas de aprendizaje con las que se han obtenido favorables resultados y que han servido como base para el desarrollo del presente método docente, es encomiable la labor que se lleva a cabo por el docente para innovar y la inversión de tiempo que conlleva no solo el planteamiento, sino también poner en práctica cada uno de los complementos de aprendizaje expuestos, ahora bien, sí que es necesario hacer hincapié en que el docente debe acompañar al alumno en cada paso formativo y si desde el primer momento demuestra empatía y una relación cercana, conllevará que las barreras entre alumno y docente no existan y la implicación del estudiante será mucho mayor obteniendo el resultado de su formación autónoma acompañado de un guía que lo motivará y ayudará en la solución de todas las cuestiones que le surjan.

Por otro lado, entendiendo que la preparación del alumno ha de focalizarse en el mundo laboral al que se enfrentará, como es el ejercicio de la profesión de abogado, la búsqueda de eficacia y éxito de forma autónoma se diluye poco a poco, el compartimento de las diversas áreas del derecho o la especialización en materias concretas, genera un desconocimiento en otras disciplinas por lo que el éxito en un procedimiento se basa en el trabajo colaborativo y cooperativo, cuando la solución de un problema se analiza de forma conjunta, se obtienen varios puntos de vista y de diversas áreas, por lo que, los equipos de trabajo y la transferencia de conocimientos es básica en cualquier procedimiento, lo cual se debe tener en cuenta en la formación del alumno, evitando que lo haga en competitividad y a través de un trabajo autónomo sobrada competitividad encontrará en la búsqueda de empleo y en su propio puesto de trabajo- con lo que se debe fortalecer desde un principio un trabajo colaborativo para que desarrolle capacidades que quizás puedan marcar la diferencia en un futuro. 
Siguiendo en esa línea de preparación para su futuro profesional, no cabe mejor aprendizaje para el estudiante que dotarle de la capacidad de saber jugar con las reglas del juego basándose en la legislación y en la forma práctica en la que ésta se utiliza, es decir, que aprendan a utilizar la legislación y las bases de datos que tienen a su alcance, los contenidos teóricos de materias sustantivas que son necesarios que sean interiorizados por el estudiante deben ir acompañados de aquellos conocimientos procesales para obtener un conocimiento completo del derecho, por lo que el hecho de memorizar contenidos no les será beneficio, ni rentable para el ejercicio de la profesión, si no se han adquirido conocimientos acerca del funcionamiento y la forma de aplicación de la norma.

\section{Bibliografía.}

-ÁLVAREZ GONZÁLEZ, E.M. (2015), "Una experiencia docente aplicada en la asignatura Fundamentos de Derecho Público del grado en Criminología", DEL VALLE MEJIAS M.E. (Coord.): Experiencias en docencia superior. Madrid: ACCI.

-CENTENERA SÁNCHEZ-SECO, F. (2017), "Clara Campoamor y el valor de la Igualdad: Una propuesta docente", Revista Jurídica de Investigación e Innovación Educativa (REJIE), núm. 16, págs. 11-33 [En línea] http://www.eumed.net/rev/rejie

-CUBILLO LÓPEZ, I.J. / FERNÁNDEZ CARRIÓN, C. / DE PRADA RODRIGUEZ, M. (2011), "Utilización del cine como herramienta para la docencia del Derecho Procesal”, PICÓ I JUNOI, J. (Dir.) Aprendizaje del Derecho Procesal: Nuevos retos de la enseñanza universitaria. Barcelona: J. M. Bosch.

-DE LUCCHI LÓPEZ-TAPIA, Y. / ROBLES GARZÓN, J. A. (2011) “Experiencias desde el aula judicial de la Facultad de Derecho de la Universidad de Málaga". PICÓ I JUNOI, J. (Dir.) Aprendizaje del Derecho Procesal: Nuevos retos de la enseñanza universitaria. Barcelona: J. M. Bosch.

-DE MIRANDA VAZQUEZ, C. (2015). "Propuesta de modelo para la docencia del Derecho Procesal", Revista de Educación y Derecho. Education and Law Review. núm. 12, págs. 151-167.

-DONDI, A. / NIEVA FENOLL, J. (2017) "Diálogos sobre la docencia en derecho procesal (Dialogues about Teaching Procedural Law)", Justicia: Revista de Derecho Procesal. J.B. Bosch, núm. 2, págs. 81-116.

-FERRERO BAAMONDE, X. / PÉREZ-CRUZ MARTÍN, A.J. (2011) "El cine como instrumento metodológico en la docencia del Derecho Procesal", PICÓ I JUNOI, J. (Dir.) Aprendizaje del Derecho Procesal: Nuevos retos de la enseñanza universitaria. Barcelona: J. M. Bosch. 
-GARCÍA MAGNA, D., CASTILlO RODRÍGUEZ, C., RIOS MOYANO, S., CRISTOFOL RODRÍGUEZ, C., CARRASCO SANTOS, M., RODRÍGUEZ MÉRIDA, R., GONZÁLEZ RAMÍREZ, D., PASTOR GARCÍA, I., \& GONZÁLEZ RAMÍREZ, D. (2011). "La interdisciplinariedad en la educación superior: propuesta de una guía para el diseño de juegos de rol". Education In The Knowledge Society (EKS), 12 (1), págs. 386-413. [En línea] http://revistas.usal.es/index.php/eks/article/view/7894/7937

-GONZALES PILLADO, E. (2009), "Una experiencia en la docencia del practicum de Derecho Procesal”, Revista de Formación e Innovación Educativa Universitaria. Vol.2, núm.3, págs. 151-159.

-GUTIERREZ BERLINCHES, A., DE PRADA RODRIGUEZ, M., CUBILLO LÓPEZ, I.J. (2010) "Las visitas a Juzgados como actividad práctica para la docencia del Derecho Procesal", Revista Jurídica de Investigación e Innovación Educativa (REJIE), núm.2, págs. 111-122 [En línea] http://www.eumed.net/rev/rejie

-KARINA CICERO, N. (2018) "El primer día de clases" Revista de Derecho y Educación. Education and Law review, núm. 18 [En línea]

http://revistes.ub.edu/index.php/RED/article/view/22927

-MONTERROSO CASADO, E. / ESCUTIA ROMERO, R. "La enseñanza práctica en 3D: Juicio virtual”, @ tic. revista d'innovació educativa, núm. 6, 2011, págs.46-54 [En línea] https://www.redalyc.org/articulo.oa?id=349532302006

-MORA CAPITÁN, B. (2011). "El aprendizaje del Derecho Procesal mediante simulación de juicios", PICÓ I JUNOI, J. (Dir.) Aprendizaje del Derecho Procesal: Nuevos retos de la enseñanza universitaria. Barcelona: J. M. Bosch.

-MOYA AMADOR, A. / SERRANO FALCÓN, C. /TOMÁS JIMÉNEZ, N. "Realidad jurídico laboral en el aula" (2011). ARBOR Ciencia, Pensamiento y Cultura. Vol.187. Extra 3, págs. 273-277 [En línea] http://arbor.revistas.csic.es/index.php/arbor/article/view/1439/1447

-SAIZ GARITAONANDIA, A. (2011), "Caminando hacia el aprendizaje cooperativo en la docencia del Derecho Procesal Penal", UNIVEST. Institut de Ciències de 1'Educació Josep Pallach (ICE). [En línea] https://dugi-doc.udg.edu/handle/10256/3695

-VARGAS GÓMEZ-URRUTIA, M. y M. HERRERA MOLINA, P. (2019) “¿Qué podemos aprender sobre Edith STEIN sobre la investigación y la enseñanza del Derecho?" Diario La Ley, núm. 7931.

-PÁGINAS WEB UTILIZADAS:

- https://ehrmcc.elsa.org/

- http://iccmoot.com/

- https://www.ilsa.org/

- https://johnhjacksonmoot.elsa.org/about/

- http://www.mootmadrid.es/2019/

- https://www.nuremberg-moot.de/index.php?id=283

- http://oiti.org/

- https://vismoot.pace.edu/site/scholarships 